\title{
COMPARISON OF SERUM VITAMIN A LEVELS BETWEEN NEONATES WITH CONGENITAL HEART DISEASE AND CONTROLS
}

\author{
SAEED ABTAHI ${ }^{1}$, ZAHRA MOSTAFAVIAN ${ }^{2 *}$, BABAK BEHNAM ${ }^{3}$, ZAHRA ABBASI SHAYE ${ }^{4}$
}

${ }^{1}$ Department of Pediatric Cardiology, Mashhad Branch, Islamic Azad University, Mashhad, Iran. ${ }^{2}$ Department of Community Medicine Mashhad Branch, Islamic Azad University, Mashhad, Iran. ${ }^{3}$ Medical Genetics Branch, National Human Genome Research Institute, Undiagnosed Diseases Program, Common Fund, Office of the Director, National Institutes of Health, Bethesda, Maryland, USA. ${ }^{4}$ Clinical Research \& Development Unit, Akbar Hospital, Mashhad University of Medical Sciences, Mashhad, Iran. Email: dr.mostafavian@mshdiau.ac.ir

Received: 26 April 2018, Revised and Accepted: 08 June 2018

\section{ABSTRACT}

Objective: Prevention of congenital heart disease (CHD) has been hampered by a lack of information about the known modifiable risk factors for abnormalities in cardiac development. Vitamin A plays an important role in the periods of rapid cellular growth and differentiation, especially during pregnancy. Assuming a link between Vitamin A levels and congenital malformations, hypothetical different levels of Vitamin A were evaluated in neonates with and without CHD, in this study.

Methods: In a case-control study that was conducted in 2015 in Mashhad/Iran, serum levels of Vitamin A in 30 neonates with CHD were compared to 30 healthy controls. The cases were diagnosed by echocardiography and recruited by convenience sampling. Demographic and laboratory data including age, sex, and serum Vitamin A level in each group were collected. Data analysis was done in SPSS V 20 software, and descriptive statistics, $t$-test, and analysis of covariance were used.

Results: The mean age in cases was $11 \pm 3.4$ days and in controls was $12.5 \pm 4.8$ days. A total of 18 patients (60\%) were male. In CHD patients, 10 cases $(33.3 \%)$ had cyanotic heart disease, and 20 cases (66.7\%) had non-cyanotic heart disease. The mean serum Vitamin A values in subjects $(11.54 \pm 9.56 \mu \mathrm{g} / \mathrm{dL})$ and controls $(21.84 \pm 14.3 \mu \mathrm{g} / \mathrm{dL})$ were significantly different, $(\mathrm{p}<0.05)$ and in case group was lower than the normal range.

Conclusion: There was a significant difference in serum Vitamin A values in subjects and controls. Therefore, awareness of people about the importance of this vitamin in preventing CHD in children seems necessary.

Keywords: Congenital heart disease, Vitamin A, Neonate.

(c) 2018 The Authors. Published by Innovare Academic Sciences Pvt Ltd. This is an open access article under the CC BY license (http://creativecommons. org/licenses/by/4. 0/) DOI: http://dx.doi.org/10.22159/ajpcr.2018.v11i10.26925

\section{INTRODUCTION}

Congenital heart disease (CHD) is the general term used to describe any gross structural abnormality of the heart or intrathoracic great vessels at birth, which is potentially of functional significance [1]. Genetic or environmental causes may lead to faulty embryogenesis during gestational weeks 3-8. In this period of time, major cardiovascular structures begin to function, and finally give rise to CHD [2]. Although the reported birth prevalence of CHD varies widely among epidemiological studies, the estimate of 8 per 1,000 live births is generally accepted as the best approximation [2-6].

CHD is regarded as a significant health burden as it is one of the most common reasons for health services utilization among children with chronic conditions (accounting 1\% of all pediatric hospitalization costs) [7]. Advances in diagnostic, surgical, interventional, and pharmacological approaches over the past decades have increased the survival of infants with CHD dramatically [3,8]. However, CHD is still the most frequent occurring congenital disorder [9] and the leading cause of infant deaths due to birth defects [7]. These patients suffer from the life-threatening associated complications such as pulmonary hypertension, endocarditis, arrhythmias, growth restriction, and repeated need for surgery [9-13].

The ability to reduce the mortality or morbidity of CHD is limited by the inadequate knowledge about its etiology. Only a small proportion $(10-15 \%)$ of cases with CHD can be traced to known causes. These etiologies may include chromosomal aneuploidies, defects in single genes or known environmental factors (such as Maternal diabetes mellitus and phenylketonuria) [9]. Other environmental risk factors are complex and commonly have genetic-environmental interactions [14].

Retinoic acid (RA), an active form of Vitamin A, is one of the environmental factors that may affect the embryogenesis [9]. It plays essential roles in many physiological functions including vision, immunity, cell differentiation, epithelial integrity, red blood cell production, and reproduction $[15,16]$. Retinaldehyde dehydrogenases are essential for the generation of embryonic RA from Vitamin A. RA functions opposing CYP26 members (which catabolize RA) and ligand-dependent nuclear receptors called RA receptors $\alpha, \beta$, and $\gamma$ and retinoid X receptors $\alpha, \beta$, and $\gamma[15,17]$.

Previous studies have been documented thatVitamin Amay be teratogenic in humans when consumed in large doses during pregnancy. On the other hand, other studies suggested that Vitamin A deficiency (VAD) may also be considered as a clinical risk similar to excess retinoid [18]. Furthermore, it is found that antenatal Vitamin A supplementation decrease anemia, preterm delivery, deformity, intrauterine growth retardation, preeclampsia/eclampsia, low birth weight, poor infant growth, neonatal/infant, and maternal mortalities [16]

However, the precise role of RA in the development of CHD has not been clearly defined yet. This study is designed to evaluate the serum levels of Vitamin A in neonates with CHD, which may lead to a better insight into its etiology.

\section{METHODS}

In a case-control study, serum Vitamin A levels were measured in neonates with CHD and compared with the control group. Due to there 
was not any similar study to our objective, we conducted this pilot study by recruiting 60 neonates in two groups.

The study was conducted in Mashhad during a 1-year period from April to March 2015 by convenience sampling method. The case group included 30 children with CHD referred to a heart clinic of Azad University of Mashhad. Inclusion criteria in the case group included diagnosis of CHD by a pediatric cardiologist and through echocardiography, age of the child from birth to 30 days old, and parents' consent for participation of their kids in the study. Exclusion criteria were low birth weight $(<2500$ g), any non-cardiac congenital disease, a known concurrent systemic disease, malnutrition, family history of heart disease, family history of congenital heart failure, and exposure to ionization radiation during pregnancy and familial marriage. Control group of 30 children was selected among children under the age of 1 month who were referred to health centers for routine childhood care by convenience sampling based on the same inclusion and exclusion criteria for the case group except having CHD. Roll out of CHD in control group was done by echocardiography similar to case group.

Demographic and laboratory data including age, sex, and serum Vitamin A level in each group were collected utilizing a checklist. In both case and control groups, $2 \mathrm{~mL}$ blood was taken from peripheral veins to separate the serum or plasma through centrifuge at $4^{\circ} \mathrm{C}$, and frozen immediately in the plastic vials. The samples were kept away from the light in chilled tubes, and the serum level of Vitamin A was measured as $\mu \mathrm{g} / \mathrm{dL}$ using HPLC liquid chromatography in one laboratory. Desired concentrations are approximately $20-60 \mu \mathrm{g} / \mathrm{dL}$. Concentrations $<20 \mu \mathrm{g} / \mathrm{dL}$ indicate deficiency [19].

The study has been approved by the University's Institutional Review Board and ethical committee with the code number IR. Neyshabur. REC. 1396. 11. The protocol was in accordance with the ethical principles of the Helsinki Declaration, and informed consent has been received from all parents and guardians of the study individuals. All costs of laboratory tests and traveling were covered by the researcher. All data and information remain confidential.

For analysis of the data, SPSS V 20 software was used. Descriptive statistics are presented for the study populations that result for continuous variables provided as means with SDs and results for categorical variables were provided as percentages. To compare the level of Vitamin A in both the case and control groups, first KolmogorovSmirnov test was done, and then they were compared using $t$-test in two independent groups. Chi-square test was used to compare qualitative variables. The significance level $<5 \%$ was considered for each test.

\section{RESULTS}

The mean age of the patients in the case and control groups was $11 \pm 3.4$ and $12.5 \pm 4.8$ days, respectively, and the average age of their mothers was $30.21 \pm 17.34$ and $28.42 \pm 15.73$ in case and control group. We did not have a significant difference between the case and control group in the age of neonates $(\mathrm{p}=0.21)$ and their mothers $(\mathrm{p}=0.08)$. Gender distribution was similar in both case and control groups so that in both groups, $18(60 \%)$ were boys and $12(40 \%)$ girls. Among children in the case group, 10 (33.3\%) and 20 (66.7\%) had cyanotic and non-cyanotic heart diseases, respectively. The mean serum Vitamin A level in children with CHD was $11.54 \pm 9.56 \mu \mathrm{g} / \mathrm{dL}$ versus $21.84 \pm 14.3 \mu \mathrm{g} / \mathrm{dL}$ in control group; which the difference was statistically significant $(p=0.005)$. This means that serum levels of this vitamin were lower in the patients' group than in the control group.

Furthermore, the mean serum Vitamin A level in the patients with CHD was compared according to the type of heart disease (cyanotic versus non-cyanotic) (Table 1). As shown in Table 1, serum level of vitamin in children with and without cyanotic heart diseases is not significantly different $(p>0.05)$.

\section{DISCUSSION}

In this study, the serum levels of Vitamin A showed a considerable decrease in children with CHD compared with normal and healthy children (without CHD) and this difference was significant.

In a similar study, mean levels of serum retinol in children with CHD in Nigeria showed no significant lower amounts than children without CHD; indicating no VAD in this disease. However, the mean serum retinol values were above the value for VAD in both the subjects and controls, addressing a possible adequate dietary source of Vitamin A in the case and control groups as Nigeria has a program in fortifying foods for the infants and the main source of this nutrient in older children and adult is palm oil [20]. Vieria et al. showed that the intake of calories, fats, fiber, sodium, potassium, and Vitamin A was below the recommended levels in children with congenital cardiopathy [21].

It has been demonstrated that Vitamin A may be crucial for heart morphogenesis during pregnancy, in terms of both its deficiency and excess. Vitamin A may play a specific role to promote heart development through activating some genes' regulations in the gastrula stage [21]. In the 1950s, it was shown that maternal VAD in a rat model causes severe cardiac and great vessels anomalies during embryogenesis. Furthermore, caused hypervitaminosis A (by active form of RA) has been one of the earliest teratogenic models of heart defects [22]. In other studies, different models have been developed to address the role of RA during early development. Among them, the Vitamin A - deficient quail was proposed as an ideal model for the Vitamin A functional studies in the vertebrate embryo. More recently, the discovery of retinoid synthesis of biochemical pathways and retinoid receptors has allowed some genetic manipulations including in mouse embryos for deciphering RA function [23]. The retinoid-binding protein/STRA6 system of VAD is the only possible mechanism for cells or tissues to obtain Vitamin A and the major transport form of it in the blood. STRA6 is a membrane receptor mediates cellular uptake of retinol, and its alteration cause CHD as well as anophthalmia, mental retardation, and embryonic lethality, demonstrating the critical function of Vitamin A in the pathogenesis of CHD [24].

In the field of human studies, Botto et al. reported that women who used multivitamin supplements in the periconceptional period were at significantly lower risk of having babies with some CHDs (specifically outflow tract and VSD) than women who not used. The timing of multivitamin use seem to be critical, as the apparent risk reduction is evident for the time of conception or early in the $1^{\text {st }}$ month but not later in the pregnancy [25].

\section{CONCLUSIONS}

Considering the results of this study and lower serum Vitamin A levels in children with CHD, awareness of people about the importance of this vitamin in preventing CHD in children seems necessary. Furthermore, family members of these children should receive counseling on how

Table 1: Distribution of serum Vitamin A levels in affected children by type of CHD

\begin{tabular}{lllllll}
\hline CHD & No & Mean+SD & Median & Range & Statistic \\
\cline { 3 - 5 } & & & Max value & Min & 13.05 \\
\hline Cyanotic & 7 & $6.08 \pm 4.9$ & 3.47 & 1.66 & $\mathrm{t}=-1.38$ \\
Non-cyanotic & 13 & $14.67 \pm 11.88$ & 9.41 & 1.97 & 60.89 & \\
\hline
\end{tabular}

CHD: Congenital heart disease 
to supplement the calories, vitamins, and minerals required when there are deficiencies that cannot be corrected through natural oral feeding, so as not to limit the synthesis necessary for the growth and development of these children.

\section{ACKNOWLEDGMENTS}

We thank the fathers and mothers of children participating in this project.

\section{REFERENCES}

1. Wan BA, Bodha RH, Wani AH. Nutritional and medicinal importance of mushrooms. J Med Plants Res 2010;4 Suppl 24:2598-604.

2. Gan CH, Nurul-Amira B, Asmah R. Antioxidant analysis of different types of edible mushrooms (Agaricus bisporous and Agaricus brasiliensis). Int Food Res J 2013;20 Suppl 3:1095-102.

3. Kumari M, Giridhar S, Khanna PK. In vitro and in vivo antioxidant potentials of Pleurotus florida in experimental animals. Mushroom Res 2004;13 Supp 1:21-6.

4. Halliwell B, Gutteridge J. Free Radicals in Biology and Medicine. $4^{\text {th }}$ ed. Oxford, UK: Oxford University Press; 2007.

5. Sheikh IA, Vyas D, Lone R, Singh V. Ganoderma lucidum and Trametes hirsute as potent antioxidant in free radical system in in vitro. World $\mathrm{J}$ Pharm Pharm Sci 2000;4 Suppl 5:1695-710.

6. Olajire A, Azeez L. Total antioxidant activity, phenolic, flavonoid and ascorbic acid contents of Nigerian vegetables. Afr J Food Sci Tech 2011;2 Suppl 2:22-9

7. Brewer MS. Natural antioxidants: Sources, compounds, mechanism of action, and potential applications. Comp Rev Food Sci Food Saf 2011;10:221-47.

8. Venkatesh R, Sood D. Review of the Physiological Implications of Antioxidants in Food Interactive Qualifying. Worcester, MA, USA: Project Report; Faculty of the Worcester Polytechnic Institute; 2009. p. 1-72.

9. Zhang YJ, Gan RY, Li S, Zhou Y, Li AN, Xu DP, et al. Antioxidant phytochemicals for the prevention and treatment of chronic diseases. Molecules 2015;20:21138-56.

10. Rajesh K, Dhanasekaran D, Panneerselvam A. Isolation and taxonomic characterization of medicinal mushroom Ganoderma spp. Acad J Micro Res 2014;2 Suppl 2:61-70.

11. Moncalvo JM, Wang H, Hseu RS. Gene phylogeny of the Ganoderma lucidum complex based on ribosomal DNA sequences. Comparison with traditional taxonomic characters. Mycol Res 1995;99:1489-99.

12. Hung PV, Morita N. Distribution of phenolic compounds in the graded flours milled from whole buckwheat grains and their antioxidant capacities. Food Chem 2009;109:325-31.

13. Tibuhwa DD. A comparative study of antioxidant activities between fresh and dry mushrooms in the genera Cantharellus and Afrocantharellus from Tanzania. Food Nut Sci 2014;5:212-21.

14. Sheikh IA, Vyas D, Ganaie MA, Dehariya K, Singh V. HPLC determination of phenolics and free radical scavenging activity of ethanolic extracts of two Polypore mushrooms. Int J Pharma Pharma Sci 2014;6 Suppl 1:679-84.

15. Khaled-khodjaa N, Boulekbache-Makhloufb L, Madani K. Phytochemical screening of antioxidant and antibacterial activities of some Laminaceae. Ind Cr Pro 2014;61:41-8.

16. Jose S, Radhamany PM. In vitro antioxidant activities, total phenolics and flavonoid of wild edible mushroom Macrolepiota mastoidea (fr.) singer. Int J Pharm Pharm Sci 2013;5 Suppl 2:161-6.

17. Russell R, Paterson M. Ganoderma-a therapeutic fungal factory phytochemistry. J Phytochem 2006;67:1985-2001.

18. Hussein JM, Tibuhwa DD, Mshandete AM, Kivaisi AK. Antioxidant properties of seven wild edible mushrooms. J Appl Microb 2015;9 Suppl 9:471-9.

19. Huang LC. Antioxidant Properties and Polysaccharide Composition Analysis of Antrodia camphorate and Agaricus blazei. Master's Thesis, National Chung-Hsing University, Taichung, Taiwan; 2000.

20. Kamiyama M, Horiuchi M, Umano K, Kondo K, Otsuka Y, Shibamoto T. Antioxidant/anti-inflammatory activities and chemical composition of extracts from the mushroom Trametes versicolor. Int J Nutr Food Sci 2013;2 Suppl 2:85-91

21. Mau JL, Lin HC, Chen CC. Antioxidant properties of speciality medicinal mushrooms. J Agric Food Chem 2002a;50:6072-7.

22. Ray G, Hussain SA. Oxidants and antioxidants and carcinogenesis. Indian J Exp Biol 2002;40:1214.

23. Soniamol J, Baby S, Varughese G, Thozhuthumparambal PS, Kainoor KJ. Antioxidative and antiinflammatory activities of the chloroform extract of Ganoderma lucidum. Ind Sci Pharm 2009;77:111-21.

24. Pal J, Ganguly S, Tahsin KS, Acharya K. In vitro free radical scavenging activity of wild edible mushroom, Pleurotus squarrosulus (Mont.) Singer. Ind J Exp Bio 2010;47:1210-8

25. Park YS, Heo BG, Ham KS, Kang SG, Gorinstein S. Analytical determination of bioactive compounds as an indication of fruit quality. JAOAC 2012;95 Suppl 6:1725-32. 